\title{
Evaluation of Fluid-Thermal Systems by Dynamic Data Driven Application Systems - Part II
}

\author{
D. Knight, Q. Ma, T. Rossman, and Y. Jaluria \\ Dept of Mechanical and Aerospace Engineering \\ Rutgers - The State University of New Jersey \\ New Brunswick, NJ 08903 \\ knight@soemail.rutgers.edu
}

\begin{abstract}
A Dynamic Data Driven Application Systems (DDDAS) methodology is developed for evaluation of fluid-thermal systems wherein a complete specification of the boundary conditions is not known a priori and experimental diagnostics are restricted to a limited region of the flowfield. The Closed Loop formulation of DDDAS is used whereby experiment and simulation are synergized in an iterative manner to determine the unknown boundary conditions, thereby enabling a full simulation (and hence, evaluation) of the fluid-thermal system. In this DDDAS methodology, the experiment directs the simulation and vice-versa. The DDDAS methodology is applied to a heated jet injected into a laminar boundary layer where the jet temperature and velocity are not known $a$ priori for the simulations. The DDDAS methodology accurately determines the unknown jet temperature and velocity.
\end{abstract}

\section{Introduction}

In a wide range of fluid-thermal systems, there is typically limited access to the flow domain for experimental measurements of the flowfield (e.g., pressure, species concentration, temperature and velocity). Examples of such systems include combustors, furnaces and reactors. For example, an optical fiber drawing furnace typically has an infrared sensor to monitor the temperature of the heating element at a single location [1. Consequently, the necessary boundary conditions (e.g., inflow, outflow, solid boundary, etc) for computational simulations (using a Computational Fluid Dynamics [CFD] code such as FluentC) are not completely known, and therefore a simulation cannot be performed.

The objective of this research is the development of a Dynamic Data Driven Applications Systems methodology that synergizes experiment and simulation in fluid-thermal systems to determine the unknown boundary conditions, thereby enabling a complete simulation of the fluid-thermal system.

\section{Dynamic Data Driven Application Systems}

The Dynamic Data Driven Applications Systems (DDDAS) concept was described in the DDDAS Workshop held at the National Science Foundation in 
March 2000 [2] and further described by Darema [3] (see also the DDDAS webpage 4). DDDAS is a unique approach to engineering and scientific research wherein experiment and simulation interact in a synergistic, symbiotic manner. There are two different implementations of DDDAS: Open Loop and Closed Loop. In Open Loop, experimental data is streamed into the simulation (or vice-versa) to achieve greater accuracy, detail and/or robustness. An example is the Dynamic Data Driven Wildfire Modeling methodology of Mandel et al [5] and Douglas et al 6]. In Closed Loop, experiment and simulation interact in an iterative manner, i.e., the experiment guides the simulation and the simulation in turn guides the experiment [3. An example is the Dynamic Data Driven Optimization Methodology (DDDOM) developed by Knight et al [78].

\section{Description of Research}

\subsection{Objective}

The objective is the development of a DDDAS methodology for synergizing experiment and simulation to evaluate fluid-thermal systems wherein the experimental measurements are restricted in region and scope, and the a priori boundary conditions for simulation are incomplete. The Closed Loop DDDAS concept is used wherein the experiment directs the simulation and vice-versa in an iterative manner. The strategy is to approximate the unknown boundary conditions by minimizing the error in the prediction of the measured data (i.e., the experiment driving the simulation), and to identify needed subsequent experimental measurements to reduce the error (i.e., the simulation driving the experiment) and also subsequent additional simulations to reduce the error (i.e., the experiment driving the simulation).

\subsection{Configuration}

We consider a rectangular jet injected perpendicular to an incompressible laminar boundary layer. The configuration is shown in Fig. 1. The inflow is an equilibrium laminar boundary layer in air defined by the specified freestream conditions (velocity $U_{\infty}$, static pressure $p_{\infty}$ and static temperature $T_{\infty}$ ) and boundary layer thickness $\delta_{\infty}$ (i.e., the laminar boundary layer thickness that would exist at the location of the jet exit in the absence of the jet). The jet is defined by the jet average velocity $U_{j}$, static pressure $p_{j}$, and static temperature $T_{j}$. The computational domain ABCDE is shown. For the simulations, the freestream conditions (velocity $U_{\infty}$, static pressure $p_{\infty}$, static temperature $T_{\infty}$ and boundary layer thickness $\delta_{\infty}$ ) are assumed known. By analogy to the optical fiber furnace, the jet average velocity $U_{j}$ and static temperature $T_{j}$ are assumed unknown (insofar as the simulations are concerned), while the jet exit pressure $p_{j}$ is assumed known. The objective is the determination of the jet average velocity $U_{j}$ and static temperature $T_{j}$ based upon a Closed Loop DDDAS methodology. The range of values for $U_{j}$ and $T_{j}$ provided to the DDDAS methdology are indicated in Table 1 (see Section 3.7). 


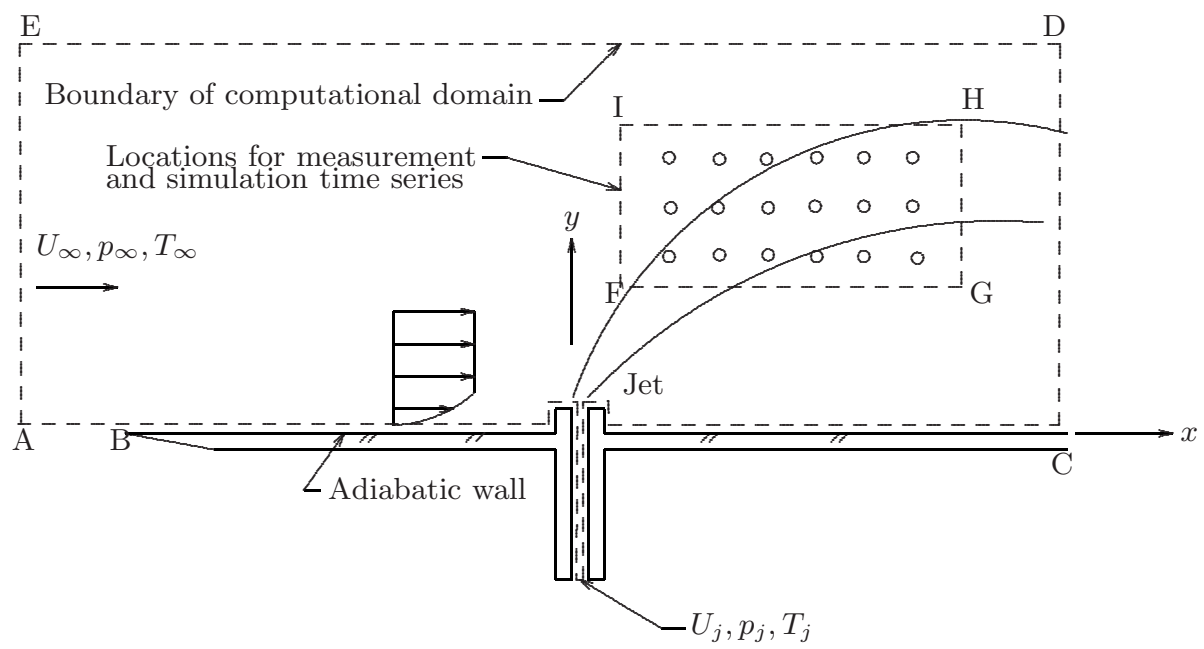

Fig. 1. Flow configuration

\subsection{Experiments}

The experiments were performed in the Rutgers Low Speed Wind Tunnel. The experimental configuration is shown in Fig. 2a. A two-dimensional slot jet protrudes from a flat plate with the jet centerline at a distance of $188 \mathrm{~mm}$ from the leading edge of the plate. The flat plate has a sharp leading edge and is raised $50 \mathrm{~mm}$ above the wind tunnel floor to avoid the tunnel floor boundary layer. The jet slot width is $3.2 \mathrm{~mm}$ and the spanwise depth is $545 \mathrm{~mm}$. The jet fences protrude $8.8 \mathrm{~mm}$ above the flat plate and each fence is $6.4 \mathrm{~mm}$ wide in the $x$-direction. The jet exit temperature was measured by a small bead thermocouple at the jet exit and was observed to vary less than $2 \%$ across the jet exit. Additional details are presented in Knight et al [9].

A diode laser system was used to measure the time-varying absorbance across the flowfield in the spanwise direction at selected locations. The measured laser absorbance is related to the local thermodynamic conditions and gas concentrations through the spectral absorption coefficient and Beer's law

$$
A=1-\frac{I}{I_{o}}=1-\exp \left(-k_{v} L\right)
$$

where $k_{v}=S \phi P, S$ is the temperature dependent linestrength $\left(\mathrm{cm}^{2}-\mathrm{cm}^{-1}\right), \phi$ is the lineshape function $\left(1 / \mathrm{cm}^{-1}\right), P$ is the partial pressure of the absorbing species given in terms of number density $\left(\mathrm{cm}^{-3}\right)$, and $L$ is the path length $(\mathrm{cm})$. The spectrally-dependent absorbance was converted from the time domain to the wavelength domain using laser calibration tuning curves. The current injection versus wavelength tuning was determined using an optical spectrum analyzer prior to performing the experiments. The spectrally-dependent absorbance $\left(k_{n}\right)$ can be integrated versus wavelength to remove the effect of the lineshape 


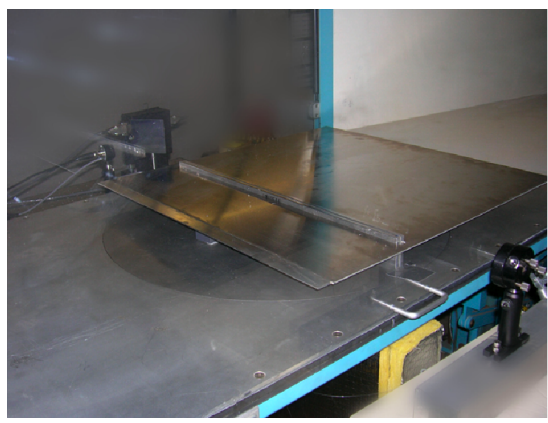

(a) Wind tunnel model

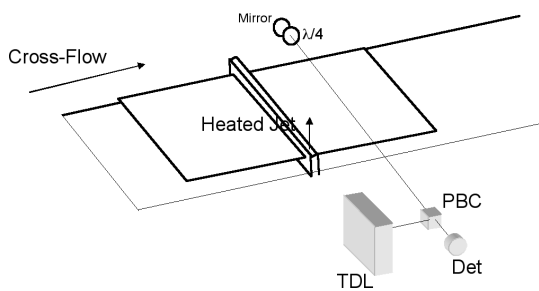

(b) Diode laser configuration

Fig. 2. Experiment

function. The integrated absorbance $(k)$ only depends on the linestrength and number density of absorbers. The temperature dependency of both quantities is known, and therefore the path-averaged temperature can be obtained from the absorption data. Further details are presented in Ma et al [10].

\subsection{Simulations}

The two-dimensional, laminar, unsteady Navier-Stokes equations were solved using Fluent ${ }^{\complement}$. The fluid is air. The Incompressible Ideal Gas Law was used together with the Boussinesq approximation. The molecular dynamic viscosity was modeled using Sutherland's Law. The simulations are second-order accurate in space and time. The spatial reconstruction is 3rd order MUSCL, and the temporal integration is Implicit Dual-Time Stepping. Twenty inner time steps at a specified inner Courant number of five are used per outer (physical) time step. A constant outer timestep $\Delta t=4 \cdot 10^{-4}$ sec was used for all simulations corresponding to an outer Courant number $C F L=\Delta t U_{\infty} / \Delta x_{\min }=4$ to 8 for the range $4 \mathrm{~m} / \mathrm{s} \leq U_{\infty} \leq 8 \mathrm{~m} / \mathrm{s}$ considered in this study. Further details are presented in Knight et al 9. Table 1 summarizes the range of parameters for the simulations.

\subsection{Validation}

A validation study was performed to assess the accuracy of the simulations by comparison with experiment. Details are presented in Knight et al 9]. An experiment was performed with $U_{\infty}=4 \mathrm{~m} / \mathrm{s}, T_{\infty}=299 \mathrm{~K}, p_{\infty}=101 \mathrm{kPa}$, $U_{j}=8.11 \mathrm{~m} / \mathrm{s}, T_{j}=398 \mathrm{~K}$ and $p_{j}=101 \mathrm{kPa}$. The mean temperature was measured at three locations in the flowfield downstream of the jet using the diode laser absorbance method (Section 3.3) and thermocouple. The freestream and jet pressure, temperature and velocity conditions were provided to the simulation 
using Fluent $($ C. The computed mean temperature at the three locations within the flowfield agreed with the experimental mean temperature within $10 \mathrm{~K}$. This represents also the uncertainty in the experimental measurement.

\subsection{Response Surface Models}

The energy equation is decoupled from the mass and momentum equations (neglecting variations in density and buoyancy effects), and thus the static temperature behaves as a passive scalar. The temperature field must therefore scale as $T(x, y, t)-T_{\infty}=\left(T_{j}-T_{\infty}\right) f\left(x, y, t ; U_{j}, U_{\infty}\right)$. Therefore, a quadratic Response Surface Model (RSM) for the time mean static temperature $T_{m}(x, y)$ may be constructed at a fixed position $(x, y)$ in the flowfield according to

$$
T_{m}(x, y)-T_{\infty}=\left(T_{j}-T_{\infty}\right)\left[\beta_{o}(x, y)+\beta_{1}(x, y)\left(\frac{U_{j}}{U_{\infty}}\right)+\beta_{2}(x, y)\left(\frac{U_{j}}{U_{\infty}}\right)^{2}\right]
$$

The coefficients $\beta_{i}(x, y)$ are obtained from simulations performed for a fixed value $T_{j}-T_{\infty}$ (selected from the range indicated in Table 1) and a set of $U_{j}$ selected from the range indicated in Table 1 For this study, the values $U_{j}=4$, 6 and $8 \mathrm{~m} / \mathrm{s}$ were selected. The freestream conditions $\left(U_{\infty}, T_{\infty}, p_{\infty}\right)$ and jet pressure $p_{j}$ are fixed as indicated in Table 1 .

\subsection{DDDAS Methodology}

The Closed Loop DDDAS methodology integrates experiment and simulation in a synergistic, iterative manner to achieve a complete evaluation of the fluidthermal system. There are five steps:

1. Select Monitor Locations for Simulations

A set $S_{s}$ of monitor locations for the simulations is selected. At each monitor location, a time series of the static temperature is obtained in every simulation. The number of monitor locations can be arbitrarily large and is limited only by available disk storage for the time series data. A total of eighteen monitor locations were selected (Table 2).

2. Generate Response Surface Model Based on Simulations for Fixed $\Delta T_{j}^{i}$ A fixed value of $\Delta T_{j}^{i}=T_{j}^{i}-T_{\infty}(i=1,2, \ldots)$ is chosen from within the range of values indicated in Table 1 . Simulations are performed for a set of values $U_{j}$ for the fixed $\Delta T_{j}^{i}$ and time series at each of the monitor locations in $S_{s}$ is recorded. A Response Surface Model for the mean static temperature $T_{m}$ at each monitor location is generated based upon the assumed value of $\Delta T_{j}^{i}$ and selected values of $U_{j}$ (see Section 3.6).

3. Select Monitor Locations for Experiments

A subset $S_{e}^{k}$ of the monitor locations $S_{s}$ is selected for the $k^{t h}$ experiment $(k=1,2, \ldots)$. Each experiment is performed at the same $U_{j}$ and $T_{j}$; however, the values of $U_{j}$ and $T_{j}$ are not known for any of the simulations. Note that the size of the set $S_{e}^{k}$ is small compared to $S_{s}$ due to the substantial amount of time required for the experimental measurements. 
4. Estimate Experimental Values for $T_{j}-T_{\infty}$ and $U_{j}$

The experimental mean temperatures are compared with the Response Surface Models to estimate the value of $T_{j}-T_{\infty}$ and $U_{j}$ in the experiment (see below).

5. Determine New Measurement Locations

The Response Surface Model is used to select the next set of monitor locations $S_{e}^{k+1}$ from $S_{s}$. Step No. 4 is repeated to provide a revised estimate of $T_{j}-T_{\infty}$ and $U_{j}$. Based upon the estimated value of $T_{j}-T_{\infty}$, the procedure repeats from Step 2 until convergence of the predicted values for $U_{j}$ and $T_{j}$.

Table 1. Flow Conditions

Table 2. Location of Monitors

\begin{tabular}{|c|c|c|c|c|c|c|c|c|c|c|}
\hline Parameter & Value & \multicolumn{2}{|c|}{ No. $x(\mathrm{~cm})$} & $y(\mathrm{~cm})$ & No. & $c(\mathrm{~cm})$ & $y(\mathrm{~cm})$ & No. & $x(\mathrm{~cm})$ & $y(\mathrm{~cm})$ \\
\hline$\overline{U_{\infty}(\mathrm{m} / \mathrm{s})}$ & 4.0 & 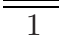 & 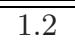 & 2.0 & $\overline{77}$ & 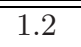 & 3.0 & 13 & 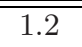 & $4 \quad 4.0$ \\
\hline$T_{\infty}(\mathrm{K})$ & 290. & 2 & 3.2 & 2.0 & 8 & 3.2 & 3.0 & 14 & 3.2 & 4.0 \\
\hline$p_{\infty}(\mathrm{kPa})$ & 101.8 & 3 & 5.2 & 2.0 & 9 & 5.2 & 3.0 & 15 & 5.2 & 4.0 \\
\hline$U_{j}(\mathrm{~m} / \mathrm{s})$ & 4.0 to 8.0 & 4 & 7.2 & 2.0 & 10 & 7.2 & 3.0 & 16 & 7.2 & 4.0 \\
\hline$T_{j}(\mathrm{~K})$ & 350 to 450 & 5 & 9.2 & 2.0 & 11 & 9.2 & 3.0 & 17 & 9.2 & 4.0 \\
\hline$p_{j}(\mathrm{kPa})$ & 101.8 & 6 & 11.2 & 2.0 & 12 & 11.2 & 3.0 & 18 & 11.2 & 4.0 \\
\hline
\end{tabular}

The estimate of the experimental values $T_{j}-T_{\infty}$ and $U_{j}$ at each step in the procedure is obtained as follows. The square error between the experimental mean temperature and the Response Surface Model for each possible subset of $l$ locations within $S_{e}^{k}$ is computed as

$$
E=\sum_{l}\left\{\Delta T_{m_{e}}-\Delta T_{j}\left[\beta_{o}(x, y)+\beta_{1}(x, y)\left(\frac{U_{j}}{U_{\infty}}\right)+\beta_{2}(x, y)\left(\frac{U_{j}}{U_{\infty}}\right)^{2}\right]\right\}^{2}
$$

where $\Delta T_{j}=T_{j}-T_{\infty}, \Delta T_{m_{e}}=T_{m_{e}}-T_{\infty}$, and the sum is over $l$ locations within $S_{e}^{k}$ (the minimum number for $l$ is 2). For example, assume $S_{e}^{k}$ contains six locations and let $l=2$. For each possible set of two locations from $S_{e}^{k}$, the values of $\Delta T_{j}$ and $U_{j}$ that minimize $E$ are determined. This yields fifteen triplets $\left(\Delta T_{j}, U_{j}, E\right)$. For a given value of $l$, the predicted values of $\Delta T_{j}$ and $U_{j}$, denoted by $\Delta T_{j}^{l}$ and $U_{j}^{l}$, are taken to be the triplet with the minimum $E$ (i.e., the values of $\Delta T_{j}$ and $U_{j}$ with the smallest square error). The procedure is repeated for all values of $l$ from $l=2$ to $n=$ size $S_{e}^{k}$. The estimate for the experimental value of $T_{j}-T_{\infty}$ is the average of these values $T_{j}-T_{\infty}=(n-1)^{-1} \sum_{l=2}^{l=n} \Delta T_{j}^{l}$ and similarly for $U_{j}$.

\subsection{Results}

The Closed Loop DDDAS methodology was applied to determine the experimental $T_{j}-T_{\infty}$ and $U_{j}$. A total of eighteen monitor locations were selected (Step 1). Response Surface Models were generated for all monitor locations for an assumed value $\Delta T_{j}=66 \mathrm{~K}$ (Step 2). Based upon these models, six locations (Nos. 
$3,9,10,14,15$ and 16) from Table 2 were selected for the experiment (Step 3). Note that the experimental $U_{j}$ and $T_{j}-T_{\infty}$ were selected by the experimentalists (Q. Ma and T. Rossman) but not communicated to the person performing the Closed Loop DDDAS Method (D. Knight) until the DDDAS method was converged. Using the experimental mean temperature measurements at the six locations, the estimated values $\Delta T_{j}=110 \pm 16 \mathrm{~K}$ and $U_{j}=7.3 \pm 1 \mathrm{~m} / \mathrm{s}$ were obtained using the Response Surface Models (Step 4). An additional set of locations for experiments was defined based upon the Response Surface Models (Nos. 2, 4, 5 and 17) (Step 5). A revised estimate $\Delta T_{j}=120 \pm 16 \mathrm{~K}$ and $U_{j}=7.1 \pm 1 \mathrm{~m} / \mathrm{s}$ were obtained using the Response Surface Models (Step 4). A revised $T_{j}-T_{\infty}=115 \mathrm{~K}$ was selected for creation of the Response Surface Models (Step No. 2) recognizing that the value originally used $\left(T_{j}-T_{\infty}=66 \mathrm{~K}\right)$ was significantly below the value predicted by the Response Surface Models. Steps 4 and 5 were repeated using the new Response Surface Models yielding the estimate $T_{j}-T_{\infty}=105 \pm 13 \mathrm{~K}$ and $U_{j}=7.1 \pm 1 \mathrm{~m} / \mathrm{s}$. The actual experimental values are $T_{j}-T_{\infty}=107 \pm 10 \mathrm{~K}$ and $U_{j}=8.0 \mathrm{~m} / \mathrm{s}$. The predicted values for $T_{j}-T_{\infty}$ and $U_{j}$ thus agree with the experimental measurements to within the experimental uncertainty (Section [3.5), thereby validating the Closed Loop DDDAS methdology.

Table 3. Results of DDDAS Method

\begin{tabular}{ccc} 
& \multicolumn{2}{c}{ Predicted } \\
Iteration & $T_{j}-T_{\infty}(\mathrm{K})$ & $U_{j}(\mathrm{~m} / \mathrm{s})$ \\
\hline \hline 1 & $110 \pm 16$ & $7.3 \pm 1$ \\
2 & $120 \pm 16$ & $7.1 \pm 1$ \\
3 & $105 \pm 13$ & $7.1 \pm 1$ \\
\hline Exp & $107 \pm 10$ & 8
\end{tabular}

\section{Conclusions}

A methodology for evaluation of fluid-thermal systems is developed based upon the Dynamic Data Driven Application Systems approach. The methodology is intended for fluid-thermal systems where complete specification of the boundary conditions is not known a priori and experimental measurements are restricted to a subregion of the fluid-thermal domain. The methodology synergizes experiment and simulation in a closed-loop, iterative manner to achieve a full evaluation of the fluid-thermal system. Results are presented for the configuration of a heated jet injected into a laminar boundary layer where the jet temperature is not known a priori. The DDDAS methodology accurately predicts the unknown jet temperature and jet velocity.

\section{Acknowledgments}

The research is sponsored by the US National Science Foundation under grant CNS-0539152 (1 Oct 2005 - 30 Sept 06). The program manager is Dr. Frederica Darema. 


\section{References}

1. S. Roy Choudhury and Y. Jaluria. Practical Aspects in the Drawing of an Optical Fiber. Journal of Materials Research, 13:483-493, 1998.

2. Dynamic Data Driven Applications Systems (DDDAS) Website. National Science Foundation, http://www.nsf.gov/cise/cns/darema/dddas/index.jsp.

3. F. Darema. Dynamic Data Driven Applications Systems: A New Paradigm for Application Simulations and Measurements. In Fourth International Conference on Computational Science, pages 662-669, Berlin, 2004. Springer-Verlag.

4. Dynamic Data Driven Applications Systems (DDDAS) Homepage. http://www.dddas.org/.

5. J. Mandel, M. Chen, L. Franca, C. Johns, A. Pulhalskii, J. Coen, C. Douglas, R. Kremens, A. Vodacek and W. Zhao. A Note on Dynamic Data Driven Wildfire Modeling. In Fourth International Conference on Computational Science, pages 725-731, Berlin, 2004. Springer-Verlag.

6. C. Douglas, J. Beezley, J. Coen, D. Li, W. Li, A. Mandel, J. Mandel, G. Qin and A. Vodacek. Demonstrating the Validity of a Wildfire DDDAS. In Sixth International Conference on Computational Science, pages 522-529, Berlin, 2006. Springer-Verlag.

7. D. Knight, G. Elliott, Y. Jaluria, N. Langrana and K. Rasheed. Automated Optimal Design Using Concurrent Integrated Experiment and Simulation. AIAA Paper No. 2002-5636, 2002.

8. H. Zhao, T. Icoz, Y. Jaluria and D. Knight. Application of Data Driven Design Optimization Methodology to a Multi-Objective Design Optimization Problem. To appear, Journal of Engineering Design, 2007.

9. D. Knight, Q. Ma, T. Rossman and Y. Jaluria. Assessment of Fluid-Thermal Systems by Dynamic Data Driven Application Systems. In International Conference on Modeling and Optimization of Structures, Processes and Systems, University of Kwazulu-Natal, Durban, South Africa, January 2007.

10. Q. Ma, Y. Luo, T. Rossman, D. Knight and Y. Jaluria. Diode Laser Measurements for DDDAS: Flowfield Reconstruction Using Dynamic Experimental and Numerical Data. AIAA Paper No. 2006-2974, 2006. 